\title{
Design and Analysis on Synchronization of a Fractional-order Bloch System
}

\author{
Xiaojun Liu \\ School of Mathematics and statistics, Tianshui Normal University, Tianshui, China \\ flybett3952@126.com
}

\begin{abstract}
In this paper, the synchronization of a fractional-order Bloch system is investigated. Based on the stability theory of fractional-order systems, the scheme of synchronization for the fractional-order complex system is given. The synchronization of the system is realized by designing the appropriate controllers. Numerical simulations are used to demonstrate the effectiveness of the proposed scheme.
\end{abstract}

Keywords: Fractional-order Bloch system, Synchronization, Chaos.

\section{Introduction}

Fractional calculus is very important in mathematical modeling. As the research of fractional calculus moves along, many nonlinear systems with fractional orders are proposed and investigated. The chaos and bifurcations which are observed in integer-order systems are also found in fractional-order ones [1-5]. It is well known that the Bloch system is very important for interpretation of the underlying the physical process of nuclear magnetic resonance. It is well known that synchronization was proposed in 1990 [6]. Nowadays, synchronization of integer-order systems has been studied extensively and several methods are extended to synchronize fractional-order systems [7-9].

\section{The fractional-order Bloch system}

The Bloch system is usually used to describe an ensemble of spins. The integer-order and fractional-order Bloch systems were studied in [10], which can be described by the following differential equations

$$
\left\{\begin{array}{l}
D^{q_{1}} x=\delta y+\gamma z(x \sin (c)-y \cos (c))-\frac{x}{\Gamma_{2}} \\
D^{q_{2}} y=-\delta x-z+\gamma z(x \cos (c)+y \sin (c))-\frac{y}{\Gamma_{2}}, \\
D^{q_{3}} z=y-\gamma \sin (c)\left(x^{2}+y^{2}\right)-\frac{z-1}{\Gamma_{1}}
\end{array}\right.
$$

where $x, y, z$ is the state variables of the system (1), $q_{1}, q_{2}, q_{3}$ the derivative orders, and $\delta, \gamma, c, \Gamma_{1}, \Gamma_{2}$ the system parameters. When the orders $q_{1}=q_{2}=q_{3}=q=0.99$, the system has a chaotic attractor with system parameters $\delta=1.26, \gamma=10, c=0.7764, \Gamma_{1}=0.5, \Gamma_{2}=0.25$, which is plotted in Fig.1. The initial conditions for the numerical simulation are $x(0)=0.1, y(0)=0.1, z(0)=0.1$ and also used in the rest of the paper. 


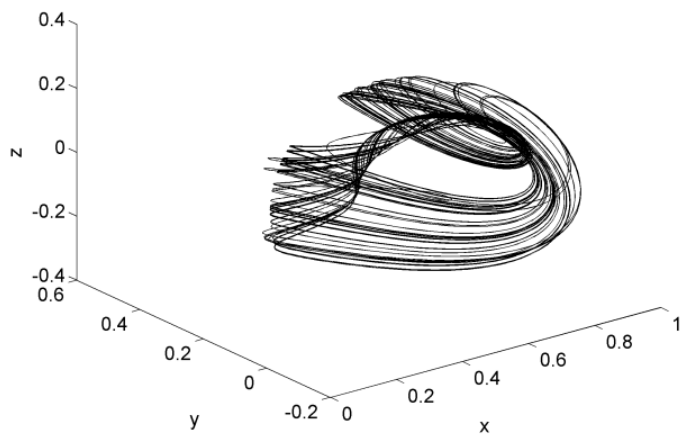

Fig.1. The chaotic attractors of the system (1)

\section{Synchronization}

In this section, the adaptive synchronization for the fractional-order Bloch system with unknown parameters will be studied.

For simplicity, the system (1) is taken as the drive system, and can be rewritten as follows:

$$
\left\{\begin{array}{l}
D^{q} x_{1}=\delta y_{1}+\gamma z_{1}\left(x_{1} \sin (c)-y_{1} \cos (c)\right)-\frac{x_{1}}{\Gamma_{2}} \\
D^{q} y_{1}=-\delta x_{1}-z_{1}+\gamma z_{1}\left(x_{1} \cos (c)+y_{1} \sin (c)\right)-\frac{y_{1}}{\Gamma_{2}}, \\
D^{q} z_{1}=y_{1}-\gamma \sin (c)\left(x_{1}^{2}+y_{1}^{2}\right)-\frac{z_{1}-1}{\Gamma_{1}}
\end{array}\right.
$$

where $\delta$ and $\gamma$ are unkonwn parameters.

The response system is described by the following differential equations

$$
\left\{\begin{array}{l}
D^{q} x_{2}=\delta y_{2}+\gamma z_{2}\left(x_{2} \sin (c)-y_{2} \cos (c)\right)-\frac{x_{2}}{\Gamma_{2}}+u_{1} \\
D^{q} y_{2}=-\delta x_{2}-z_{2}+\gamma z_{2}\left(x_{2} \cos (c)+y_{2} \sin (c)\right)-\frac{y_{2}}{\Gamma_{2}}+u_{2} \\
D^{q} z_{2}=y_{2}-\gamma \sin (c)\left(x_{2}{ }^{2}+y_{2}{ }^{2}\right)-\frac{z_{2}-1}{\Gamma_{1}}+u_{3}
\end{array}\right.
$$

where $u_{1}, u_{2}, u_{3}$ the synchronization controllers needed to be designed, $8 /, 9 /$ are the estimations of the uncertain parameters. The synchronization error variables are defined as $e_{1}=x_{2}-x_{1}, e_{2}=y_{2}-y_{1}, e_{3}=z_{2}-z_{1} \quad, \quad$ and the estimation errors of unknown parameters $e_{\delta}=\delta-\delta, e_{\gamma}=\gamma-\gamma$. By subtracting the system (2) from the (3), we can obtain the error dynamical system, which is given as follows: 


$$
\left\{\begin{aligned}
D^{q} e_{1}= & \delta e_{2}+y_{1} e_{\delta}+\gamma e_{3}\left(x_{2} \sin (c)-y_{2} \cos (c)\right)+\gamma z_{1}\left(e_{1} \sin (c)-e_{2} \cos (c)\right) \\
& +e_{\gamma} z_{1}\left(x_{1} \sin (c)-y_{1} \cos (c)\right)-\frac{e_{1}}{\Gamma_{2}}+u_{1} \\
D^{q} e_{2}= & -\delta e_{1}-x_{1} e_{\delta}-e_{3}+\gamma e_{3}\left(x_{2} \cos (c)+y_{2} \sin (c)\right)+\gamma z_{1}\left(e_{1} \cos (c)+e_{2} \sin (c)\right) \\
& +e_{\gamma} z_{1}\left(x_{1} \cos (c)+y_{1} \sin (c)\right)-\frac{e_{2}}{\Gamma_{2}}+u_{2} \\
D^{q} e_{3}= & e_{2}-\gamma\left(e_{1}\left(x_{1}+x_{2}\right)+e_{2}\left(y_{1}+y_{2}\right)\right) \sin (c)-e_{\gamma}\left(x_{1}^{2}+y_{1}^{2}\right) \sin (c)-\frac{e_{3}}{\Gamma_{1}}+u_{3}
\end{aligned}\right.
$$

In order to realize the synchronization of the drive and response systems, the controllers should be designed properly. Therefore, the following controllers and laws of the uncertain parameters are presented to ensure the system (2) effectively synchronizes the system (3).

The controllers and laws of the uncertain parameter are designed as follows:

$$
\begin{aligned}
& \left\{\begin{array}{l}
u_{1}=-\delta e_{2}-\gamma e_{3}\left(x_{2} \sin (c)-y_{2} \cos (c)\right)-\gamma z_{1}\left(e_{1} \sin (c)-e_{2} \cos (c)\right)+\left(\frac{1}{\Gamma_{2}}-1\right) e_{1} \\
u_{2}=\delta e_{1}+e_{3}-\gamma e_{3}\left(x_{2} \cos (c)+y_{2} \sin (c)\right)-\gamma z_{1}\left(e_{1} \cos (c)+e_{2} \sin (c)\right)+\left(\frac{1}{\Gamma_{2}}-1\right) e_{2} \\
u_{3}=-e_{2}+\gamma\left(e_{1}\left(x_{1}+x_{2}\right)+e_{2}\left(y_{1}+y_{2}\right)\right) \sin (c)+\left(\frac{1}{\Gamma_{1}}-1\right) e_{3}
\end{array}\right. \\
& \left\{\begin{array}{l}
D^{q} \delta=-y_{1} e_{1}+x_{1} e_{2} \\
D^{q} \gamma=-z_{1}\left(x_{1} \sin (c)-y_{1} \cos (c)\right) e_{1}-z_{1}\left(x_{1} \cos (c)+y_{1} \sin (c)\right) e_{2}+\left(x_{1}^{2}+y_{1}^{2}\right) \sin (c) e_{3}
\end{array}\right.
\end{aligned}
$$

In numerical simulations, the real values of the unknown parameters are $\delta=1.26, \gamma=10$ when $c=0.7764, \Gamma_{1}=0.5, \Gamma_{2}=0.25, q=0.98$. The initial conditions of the drive and response systems are $(0.1,0.1,0.1)$ and $(1,2,3)$, respectively. The synchronization results of the numerical simulation are depicted in Fig.2. From which it can seen that the error variables tend to 0, and the estimations of uncertain parameters converge to their real values. These results demonstrate the effectiveness of the synchronization controllers and laws of unknown parameters.
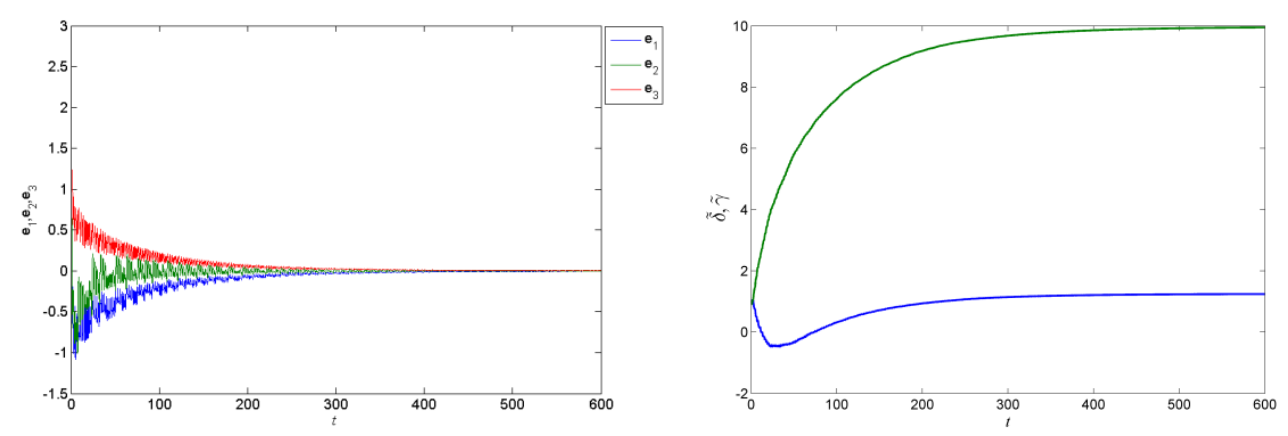

$\begin{array}{ll}\text { (a) The synchronization error curves } & \text { (b) The identification curves of the unknown parameters }\end{array}$

Fig. 2 The synchronization results of the numerical simulation

In this paper, synchronization of a fractional-order Bloch system is investigated. Based on the stability theory of fractional-order systems, the scheme of synchronization for the fractional-order 
complex system is proposed. The synchronization for the system is realized by designing appropriate controllers. Numerical simulations are used to demonstrate the effectiveness of the proposed scheme.

\section{References}

[1] T.T. Hartley, C.F. Lorenzo, and H.K. Qammer: Chaos in a fractional order Chua's system. Circuits \& Systems I Fundamental Theory \& Applications IEEE Transactions on, 42(1995): 485-490.

[2] Z.M. Ge, and C.Y. Ou: Chaos in a fractional order modified Duffing system. Chaos, Solitons and Fractals, 34(2007): 262-291.

[3] I. Grigorenko, and E. Grigorenko: Chaotic dynamics of the fractional Lorenz system. Physical Review Letters, 91(2003): 034101.

[4] J.G. Lu, Chaotic dynamics of the fractional-order Ikeda delay system and it synchronization. Physics Letters A, 59(2006):301-305.

[5] V. Daftardar-Gejji, and S. Bhalekar, Chaos in fractional ordered Liu system. Computers \& Mathematics with Applications, 59(2010): 1117 -1127.

[6] L.M. Pecora, and T.L. Carroll: Synchronization in chaotic systems. Physical Review Letter, 64(1990): 821-841.

[7] G.M. Mahmoud, T. Bountis, G.M. Abdel-Latif, and E. E. Mahmoud: Chaos synchronization of two different chaotic complex Chen and Lü systems. Nonlinear Dynamics, 55(2009): 43-53.

[8] G.M. Mahmoud, and E.E. Mahmoud: Complete synchronization of chaotic complex nonlinear systems with uncertain parameters. Nonlinear Dynamics, 62(2010): 875-882.

[9] G.M. Mahmoud, and E.E. Mahmoud: Synchronization and control of hyperchaotic complex Lorenz system. Mathematics and Computers in Simulation, 80(2010)2286-2296.

[10] H. Nasr-eddine, and H. Tarek: Chaotic dynamics of the fractional order nonlinear Bloch system. Electronic Journal of Theoretical Physics, 8(2011), 1-12. 